\title{
Challenges in the Development of Novel Targeted Therapies
}

\author{
Gottfried E. Konecny \\ Department of Obstetrics and Gynecology, Division of Gynecologic Surgery, Mayo Clinic, Rochester, MN, USA
}

\section{Finding 'Drivers' not 'Passengers'}

Breast cancers are defined as clonal proliferations caused by mutations that confer a selective growth advantage on cells. In an effort to identify novel mutations of cancer genes, the Cancer Genome Project is currently performing a systematic resequencing of cancer genomes for yet undiscovered somatic mutations. A recent publication of this consortium in Nature reports more than 1,000 somatic mutations in coding exons of 518 protein kinases in 210 samples of diverse human cancers [1]. Protein kinases were selected for this study as mutations of protein kinases are commonly found in cancer and because inhibitors have recently shown remarkable efficacy in cancer treatment [2]. The authors of the Cancer Genome Project Study then distinguished between two distinct groups of mutations: 'Driver' mutations which confer growth advantage on the cell in which they occur (and which are casually implicated in cancer development and therefore have been positively selected). In contrast, 'Passenger' mutations have not been subject to selection as they are biologically neutral and do not confer growth advantage. Using computer modeling, the authors discovered that only $15 \%$ of the novel protein kinase mutations were actually estimated to be 'Driver' mutations and $85 \%$ were estimated to be 'Passenger' mutations not being causally implicated in cancer development. More importantly, these 'Driver' mutations of protein kinases were implicated in about one third of cancers studied. Their paper can be considered to be a landmark study as it is the first report of the Cancer Genome Project to identify a larger number of novel cancer genes that could be utilized as targets for new kinase inhibitors. Moreover, its importance should be seen in the distinction between 'Driver' and 'Passenger' mutations. This distinction is important because failure to identify 'Drivers' in the disease process will lead to target-based treatments that are biologically neutral. Currently established targets in breast cancer treatment can be viewed in a very similar way. For example, HER2 overexpression is, without question, a 'Driver' (albeit its cause not being a mutation but rather an amplification of the wild-type gene). HER2 inhibitors have resulted in meaningful clinical responses when inhibiting HER2's biologic function as a 'Driver'. In contrast, EGFR is commonly expressed at high levels in cancer but rarely a 'Driver' of the disease process. Even if EGFR is expressed at very high levels, it is most commonly a 'Passenger' alteration without biological relevance as it is not conferring a growth advantage. In most breast cancer trials responses to EGFR inhibitors were rare, and responses did not correlate with percentage or intensity of EGFR staining. In retrospect, this result is not surprising in light of the known mechanisms for EGFR activation. Amplification of EGFR does not occur frequently in breast cancer, and neither do activating EGFR mutations. EGFR is not constitutively active in breast cancer but instead is probably subject to direct ligand activation by transforming growth factor $\alpha$, EGF, and related growth factors. Hence, EGFR overexpression is not required for EGFR-dependent tumorigenesis and tumor progression. Recent strategies to select patient subgroups, e.g. 'triple negative' breast cancer (HER2, ER, and PR negative) may help to define or enrich a patient subset where EGFR may more commonly be a 'Driver' rather than just a 'Passenger'.

\section{Signaling Redundancy}

Nevertheless, even as biomarkers indicative of disease mechanisms became targets for therapy, despite promising scientific rationale, many of these targeted agents have shown disappointing efficacy in clinical trials, or clinical studies have revealed benefit in some trials but not in others. In most cases, it remains uncertain why the agents failed. The development of

\begin{tabular}{ll}
\hline KARGER & ๑ 2007 S. Karger GmbH, Freiburg \\
Fax +49 7614520714 & Accessible online at: \\
$\begin{array}{l}\text { E-mail Information@Karger.de } \\
\text { www.karger.com }\end{array}$ & www.karger.com/brc
\end{tabular}


novel agents targeting tumor vasculature is an excellent example for these challenges. The ability to manipulate tumor vasculature represents a promising avenue for cancer therapy, and a diverse group of drugs is currently being developed to target components and pathways associated with these processes. To date, more than 100 potential angiogenesis inhibitors have been identified, and more than 30 antiangiogenic drugs are currently being tested in clinical trials. Many of these, however, have often failed to meet the promise suggested by preclinical studies. In retrospect, these disappointments may reflect incorrect identification of targets, insufficient potency of the agent(s) tested or, most importantly, redundancy of signaling and redundancy of targets. For example, angiogenesis is regulated through numerous complex pathways, and most tumors express multiple angiogenic pathways. What is not made clear in simplified schematics is that many signaling pathways appear to constitute a highly integrated network rather than a simple linear structure down a single pathway. This allows for considerable crosstalk, networking, and redundancy among pathways in cell signaling. Thus, inhibition of particular receptors known to activate certain pathways may still not result in complete or even significant inhibition of signaling or cell activation.

\section{Patient Selection}

Other problems can be illustrated by recent phase III clinical trials of bevacizumab, a recombinant humanized monoclonal antibody to VEGF, in combination with standard chemotherapy regimens. One trial in second line treatment of advanced breast cancer was assessed as a failure, whereas the trial in first line treatment of advanced breast cancer was a success. The 'negative' trial involved treatment with bevacizumab in combination with capecitabine in advanced breast cancer, whereas the 'positive' trial involved testing first line chemotherapy with paclitaxel in combination with bevacizumab as a first line therapy in metastatic breast cancer. When these trials were designed, the implicit assumption was that tumor angiogenesis in advanced breast cancer is driven predominantly by VEGF. However, it is likely that elevated levels of VEGF expression occur in only $40-50 \%$ of metastatic breast cancers. Therefore, it may have been the case that at least half the patients in the breast cancer trial who received bevacizumab could not, in retrospect, have benefited from receiving the drug. Failure to identify patients who express VEGF in a functionally significant manner could obscure an otherwise useful drug. For example, even if only $30 \%$ of breast cancers are driven by VEGF, this still represents a sizeable patient population. But clearly, strategies are needed to screen for patients whose tumors are most amenable to specific targeted agents [3].
Recent technological advances now provide an unprecedented opportunity for the development of biomarkers relevant to cancer mechanisms and patient selection. Most promising among these are molecular profiling approaches based on genomics or proteomics to define the molecular signature of cancers using patterns of gene and protein expression associated with different cancers and responses to targeted agents. Integration of these technological advances into clinical trials exploring the efficacy of novel targeted agents will secure the identification of relevant cancer mechanisms and help select those patients most likely to benefit from these targeted agents.

\section{Optimization}

Other challenges face the field of targeted therapeutics. For example, in testing new targeted therapies, do we simply integrate such therapies into established regimens? The traditional method for assessing effectiveness is to add a new agent to a regimen with established effectiveness and to compare it with the standard treatment. In the case of targeted therapies, this frequently may mean combining the targeted agent with regimens with which they do not have optimal interactions.

In addition to the large number of currently known potential targets, the recent paper published by the Cancer Genome Project Consortium revealed even more potential cancer genes that warrant further evaluation [1]. This rapid development in target discovery poses a new challenge. It is now more important than ever to establish better approaches for the preclinical and clinical evaluation of these new agents. With the large number of targeted therapies now in development, rational approaches for deciding which of the new therapies to test and which ones in combination are needed. It will be very important to develop preclinical systems and/or models capable of identifying optimal combinations prior to evaluation in the clinical setting.

This issue of BREAST CARE highlights the potential, as well as the associated challenges, of using mechanism-based biomarkers to facilitate the development of molecular targeted therapies in breast cancer. The articles of Untch and Jackisch [4], Schuetz et.al. [5], Fasching et al. [6], and Jackisch and Untch [7] demonstrate that the discovery and validation of mechanism-based biomarkers and the selection of the appropriate subset of patients in which the biomarker has functional relevance will be integral to the successful development of novel targeted agents in breast cancer. Moreover, their articles clearly emphasize that the translation of molecular insights into useful therapeutic approaches is highly complex, and the success of any particular approach is by no means guaranteed. 


\section{References}

1 Greenman C, Stephens P, Smith R, Dalgliesh GL, Hunter C, Bignell G, Davies H, Teague J, Butler A, Stevens C, Edkins S, O'Meara S, Vastrik I, Schmidt EE, Avis T, Barthorpe S, Bhamra G, Buck G, Choudhury B, Clements J, Cole J, Dicks E, Forbes S, Gray K, Halliday K, Harrison R, Hills K, Hinton J, Jenkinson A, Jones D, Menzies A, Mironenko T, Perry J, Raine K, Richardson D, Shepherd R, Small A, Tofts C, Varian J, Webb T, West S, Widaa S, Yates A, Cahill DP, Louis DN, Goldstraw P, Nicholson AG, Brasseur F, Looijenga L, Weber BL, Chiew YE, DeFazio A, Greaves MF, Green AR, Campbell P, Birney E, Easton DF, ChenevixTrench G, Tan MH, Khoo SK, Teh BT, Yuen ST, Leung SY, Wooster R, Futreal PA, Stratton MR: Patterns of somatic mutation in human cancer genomes.Nature 2007;446(7132):153-8.

2 Park JW, Kerbel RS, Kelloff GJ, Barrett JC, Chabner BA, Parkinson DR, Peck J, Ruddon RW, Sigman CC, Slamon DJ: Rationale for biomarkers and surrogate end points in mechanism-driven oncology drug development. Clin Cancer Res 2004;10(11): 3885-96.

3 Baselga J: Targeting tyrosine kinases in cancer: the second wave. Science 2006;312(5777):1175-8.

4 Untch M, Jackisch C: Therapy with lapatinib: current status in women with advanced breast cancer. Breast Care 2007;2(2):76-80.

5 Schuetz F, Sohn C, Schneeweiss A: Bevacizumab in the treatment of metastatic breast cancer. Breast Care 2007;2(2):82-88.

6 Fasching PA, Lux MP, Rauh C, Jud S, Beckmann MW: Clinical and preclinical experience with gefitinib and sunitinib. Breast Care 2007;2(2): 68-73.

7 Jackisch C, Untch M: Trastuzumab in the treatment of breast cancer - new developments. Breast Care 2007;2(2):90-98. 